\title{
Intensive short-term vasodilation effect in the pain area of sciatica patients - case study
}

\author{
Elżbieta Skorupska ${ }^{1 *}$, Michał Rychlik², Wiktoria Pawelec ${ }^{3}$, Agata Bednarek ${ }^{1}$ and Włodzimierz Samborski ${ }^{1}$
}

\begin{abstract}
Background: Varied and complicated etiology of low back pain radiating distally to the extremities is still causing disagreement and controversy around the issue of its diagnosis and treatment. Most clinicians believe that the source of that pain is generally radicular. While some of them postulate the clinical significance of the sacroiliac joint syndrome, others demonstrate that almost one in five people with back pain experience symptoms indicative of the neuropathic pain component. To date, neuropathic involvement has not been completely understood, and different mechanisms are thought to play an important role. It has been established that muscle pain (myofascial pain) e.g. active trigger points from the gluteus minimus, can mimic pain similar to sciatica, especially in the chronic stage. This paper describes patients presenting with radicular sciatica (case one and two) and sciatica-like symptoms (case three). For the first time, intensive short-term vasodilation in the pain area following needle infiltration of the gluteus minimus trigger point was recorded.

Case presentation: Three Caucasian, European women suffering from radicular sciatica (case one and two) and sciatica-like symptoms (case three) at the age of 57, 49 and 47 respectively underwent infrared camera observation during needle infiltration of the gluteus minimus trigger point. The patients were diagnosed by a neurologist; they underwent magnetic resonance imaging, electromyography, neurography and blood test analysis. Apart from that, the patients were diagnosed by a clinician specializing in myofascial pain diagnosis.

Conclusion: In the examined cases, trigger points-related short-term vasodilation was recorded. Confirmation of these findings in a controlled, blinded study would indicate the existence of a link between the pain of sciatica patients (radicular or sciatica-like pain) and the activity of the autonomic nervous system. Further studies on a bigger group of patients are still needed.
\end{abstract}

Keywords: Sciatica, Trigger points, Infrared camera, Vasodilation, Autonomic phenomenon

\section{Background}

Varied and complicated etiology of low back pain radiating distally to the extremities is still causing disagreement and controversies around the issue of its diagnosis and treatment. Most clinicians believe that the source of that pain is generally radicular. While some of them postulate the clinical significance of the sacroiliac joint syndrome [1], others demonstrate that almost one in five people with back pain experience symptoms indicative of the neuropathic pain component. To date, neuropathic involvement has not been completely understood, and different mechanisms are thought to play an important role.

\footnotetext{
* Correspondence: skorupska@ump.edu.pl

'Department of Rheumatology and Rehabilitation, Poznan University of Medical Sciences, Poznan, Poland

Full list of author information is available at the end of the article
}

A combination of nociceptive and neuropathic paingenerating mechanism is thought to be involved, which led to the establishment of the term mixed pain syndrome [2]. It has been established that muscle pain (myofascial pain) e.g. active trigger points from the gluteus minimus, can mimic pain similar to sciatica, especially in the chronic stage [3]. Trigger points (TrPs) are hyperirritable nodules within taut bands of skeletal muscles, which can provoke sensory, motor, and autonomic symptoms. These symptoms can be released through the damage of disturbed neuromuscular junction (trigger point) by rapid, repetitive needle insertion to the trigger points called dry needling (DN), which causes muscle injury and may damage nerve fibers $[4,5]$.

The diagnosis of myofascial pain (MPS) is based on palpatory criteria defined by Simons and Travell [3]. 
Even though MPS diagnostic criteria have been precisely defined, some clinicians are still denying their existence. Nowadays, a rapid development of basic knowledge of MPS and its diagnosis is observed. As an example, trigger points have been objectively verified by means of magnetic resonance imaging [6,7] sonoelastography [8] or intramuscular electromyography [9]. Unfortunately, these techniques are not easily applicable to clinical practice at this time. For that reason, the diagnosis of MPS is still based on palpatory diagnostic criteria.

This paper describes patients presenting with chronic low back pain radiating to the left lower limb diagnosed neurologically as radicular sciatica (case one and two) and sciatica-like symptoms (case three). Additionally, patients were re-diagnosed with myofascial pain syndrome by another clinician. The examined patients presented autonomic nervous system activity, namely intensive short-term vasodilation effect in the pain area after needling infiltration of the gluteus minimus trigger point.

\section{Case presentation}

\section{Ethics Statement}

The study was conducted in accordance with the Declaration of Helsinki approved by the Ethics Committee of Poznan University of Medical Sciences (no. 630/13). All subjects gave written informed consent to participate in the study before data collection. A detailed description of all examination and treatment procedures, including dry needling (DN), and risks involved in this study has been provided to the participants. Participants had the right to refuse DN treatment and withdraw from the study at any time without penalty.

\section{Case 1}

A 57-year-old female presented to the University outpatient clinic with a twelve weeks history of progressive pain in the left lower extremity and low back pain (LBP). Her past medical history was remarkable for several prior episodes of lower back pain. She described her state as aggravating with time, her pain ranged between 3 and 8 points on the visual-analogue scale (VAS). The patient reported pain in the lower back, groin and posterolateral thigh. She complained of some temporary but severe pain in the calf and foot, especially during the night. Standard treatment for radicular pain failed (she received miorelaxants, anti-inflammatory drugs and painkillers (paracetamol, opioids). She was re-diagnosed with sciatica of radicular origin by an independent university neurologist. The diagnosis was based on detailed bedside neurological examinations and extensive neurological screening examination (NSE). Lasegue test, femoral nerve tension test, passive dorsiflexion test (Bragard test) and Lasegue test plus neck flexion were all positive, cross Lasegue sign was the only negative.
The locomotor system examination confirmed tension of the paraspinal muscles on the left side at L2/L3 and pain of the spinous processes. Although a slight muscle weakness for flexion and extension (4/5) of the thigh and knee on the right side was discovered, other parameters were normal (5/5). Results of other neurological assessments were normal. Magnetic resonance imaging (MRI) scan of the lumbar and sacral spine revealed right disc protrusion at L3-L4 indenting thecal sac and the same side disc-root conflict. No abnormalities in sacroiliac joint were found. In the electromyographical examination, (erector spinae, quadratus lumborum, gluteus maximus, rectus femoris and gastrocnemius), a normal result was found during rest. In maximum contraction, features of bilateral motor unit dysfunction in innervation of L4-L5, L5-S1 were discovered. Conduction of motor fibers in examined nerves in lower limbs was normal, as was conduction of motor fibers in ventral root L5-S1. Laboratory tests revealed leukopenia, neutropenia,

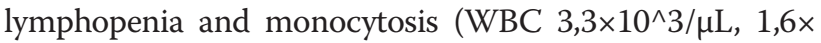
$10^{\wedge} 3 / \mu \mathrm{L}, 1,3 \times 10^{\wedge} 3 / \mu \mathrm{L}$ and $10,9 \%$ respectively, ESR and C-reactive protein (CRP) were within limits ESR -12/lh, CRP $-0.1 \mathrm{mg} / \mathrm{l}$.

\section{Case 2}

A 49-year-old female presented to the University outpatient clinic with a four weeks history of intensive pain in left lower extremity and low back pain. Medical history revealed several prior episodes of LBP which started in 2003. The patient confirmed the level of pain as 8 according to VAS. The pain covered her lower back, lateral thigh, calf and lateral edge of the foot. Apart from the pain, she also complained of numbness. Previous sciatica treatment failed (she received thiamin pyrophosphate, cobalamin, anti-inflammatory drugs and paracetamol). Based on detailed bedside neurological examinations and NSE, she was re-diagnosed with sciatica of radicular origin by an independent university neurologist. These examinations revealed abnormal gait on the heel of the left foot, slight weakness of the left foot inversion (4/5), left foot dorsiflexion (4/5), great toe dorsiflexion (4/5) and temperature dysesthesia of the lateral edge of the foot. While the examination revealed a positive Lasegue test, Bragard test and Lasegue test plus neck flexion, the rest of the tests were negative. Other results of neurological examination were normal. The following results were confirmed through MRI examination: posterior disc bulging without thecal sac compression at L2-L3, minor disc protrusion with thecal sac modelling and a disc-root conflict at L3-L4, bilateral disc-root conflict at L5, severe compression of the left nerve root and thecal sac at L5-S1, and L5-S1 disc space narrowing due to disc protrusion. No abnormalities in sacroiliac joints were found. Similarly to case one patient, electromyographic 
and electroneurographic examinations were carried out. Although their results turned out to be normal, during maximuml contraction, motor unit dysfunction features were discovered in the left side of L4-L5 innervation. Laboratory tests revealed a slight monocytosis (11\%), elevated MCV (116.3 fL) and $\mathrm{MCH}(39.5 \mathrm{pg})$. Other parameters were normal - the erythrocyte sedimentation rate (ESR) reached 4/lh, C-reactive protein levels (CRP) reached $0.1 \mathrm{mg} / \mathrm{l}$.

\section{Case three}

A 46-year-old female presented to the University outpatient clinic with a three weeks history of intensive pain in the left lower extremity and low back pain. She confirmed the level of pain on five according to VAS. Patient localised the pain in the lower back, posteriorlateral thigh and calf. She received pain killers and antiinflammation drugs from her GP. She was diagnosed by university neurologist with sciatica-like symptoms on the basis of bedside examinations and NSE (a norm for every component of the test was confirmed). Leftward disc herniation at the L5-S1 with left nerve root intracanal compression were the only abnormalities registered in MRI. In electroneurography, no dysfunction of peripheral conduction of lower limb nerves was discovered. However, by means of electromyography, features of motor unit dysfunction in L4-L5 innervation were recorded on the left side during maximal contraction. Laboratory tests confirmed neutropenia (NEUT\#1.93 $\times 10^{\wedge} 3 / \mu \mathrm{L}$; NEUT\% $33.6 \%$ ), slight monocytosis (MO\# $0.83 \times 10^{\wedge} 3 / \mu \mathrm{L} ; \mathrm{MO} \%$ $14.3 \%$ ); elevated levels of lymphocytes $49.8 \%$; and trombocytosis $863 \times 10^{\wedge} 3 / \mu \mathrm{L}$. Other results were normal - ECR 8/lh, CRP $5.3 \mathrm{mg} / \mathrm{l}$.

\section{Myofascial pain examinations}

In the patients, the presence of an active trigger point in the anterior fibres of the gluteus minimus muscle (GM) was confirmed according to Travell and Simons' criteria [3]. Within the GM, two most painful points with referred pain were marked. Then, the participants were subject to dry needling technique performed in the previously marked points under control of an infrared thermovision camera. Needling was performed with $0.30 \mathrm{~mm}$ diameter, $60 \mathrm{~mm}$ long sterile acupuncture needles SE L (Serin Corp, Shizuoka, Japan). Each needle was packed separately. The needle pierced the skin and reached the painful point with referred pain. The recognizable pain started to withdraw partially and repeatedly and eventually both the pain and muscle fiber contraction subsided. The time of needling was maximum 6 minutes for any given point. After needling of both points was conducted, further thermovisual imaging was performed. At the end, a set of thermovisual images of the final state was recorded. For the diagnostic test, a thermovision touchless camera using the 8-14 $\mu \mathrm{m}$ waveband and working in real time was applied. The camera was equipped with uncooled VOx (vanadium oxide) microbolometer. To obtain the stability of patient's body temperature and to ensure the adjustment of the recording camera's temperature to the interior conditions, the evaluation began 30 minutes after the patient had entered the examination room. Thermal isolation of the evaluated area from other thermal factors that might have influenced the evaluation, including other parts of the patient's and doctor's bodies was ensured. Moreover, when performing thermovisual imaging, the general rules of camera usage were followed.

During the evaluation, all thermovisual images were measured and recorded within the measurement range displayed by the camera and set between 25 and 45 degrees Celsius (in individual cases lower levels of temperature can be set).

\section{Results of the infrared thermovisual observation}

After dry needling of active TrPs in the gluteus minimus muscle in the pain area (sciatica), the short-term vasodilation effect was recorded (under infrared thermovision camera) (Figure 1). The increase of maximum and average temperature (Tmax, Tavr) limited to the subarea with vasodilation effect was confirmed (case $1 \Delta \mathrm{T} \max$ $+1.3^{\circ} \mathrm{C} ; \Delta \mathrm{T}$ avr $0.9^{\circ} \mathrm{C}$; case $2 \Delta \mathrm{T} \max +2.1^{\circ} \mathrm{C} ; \Delta \mathrm{T}$ avr $1.1^{\circ} \mathrm{C}$; case $3 \Delta \mathrm{T} \max +2.1^{\circ} \mathrm{C} ; \Delta \mathrm{T}$ avr $+1.8^{\circ} \mathrm{C}$ ). Subareas with the vasodilation of the highest temperature increase are shown on Figure 2. Dry needling provoked spreading of subareas (iso-areas) with the highest temperature. For case one patient, the iso-area $6.9 \mathrm{~cm}^{2}\left(35.6^{\circ} \mathrm{C}\right)$ spread to $59.9 \mathrm{~cm}^{2}$ $\left(35.6^{\circ} \mathrm{C}-36.2^{\circ} \mathrm{C}\right)$ post-needling, and in rest position six minutes later, further spreading was observed - finally the area reached $361.4 \mathrm{~cm}^{2}$ and the temperature rose to $36.9^{\circ} \mathrm{C}$, then a slow decrease of the vasodilation subarea and temperature were observed. For case two respectively: $9.1 \mathrm{~cm}^{2}\left(35.7^{\circ} \mathrm{C}\right)$ to $119.3 \mathrm{~cm}^{2}\left(36.2^{\circ} \mathrm{C}\right)$ to $232.3 \mathrm{~cm}^{2}$ $\left(36.2^{\circ} \mathrm{C}\right)$. For case three: $0.1 \mathrm{~cm}^{2}\left(34.2^{\circ} \mathrm{C}\right)$ to $508.6 \mathrm{~cm}^{2}$ $\left(34.9^{\circ} \mathrm{C}\right)$ to $635.7 \mathrm{~cm}^{2}\left(36.5^{\circ} \mathrm{C}\right)$.

In each of these cases, the first manifestations of vasodilation were recorded immediately after needle insertion to a trigger point. The vasodilation effect increased during $\mathrm{DN}$, reached its highest at approximately $6 \mathrm{mi}-$ nutes post DN and decreased afterwards. These reactions depended on pain severity during procedure and the time post-DN (Additional file 1).

\section{Discussion}

The main outcome of this study was to establish the short-term vasodilation effect in the pain area of patients diagnosed with sciatica. The reaction recorded during procedure may indicate the existence of a link between sciatica and sympathetic nerve activity, which has already been suggested [2]. Additionally, the involvement 


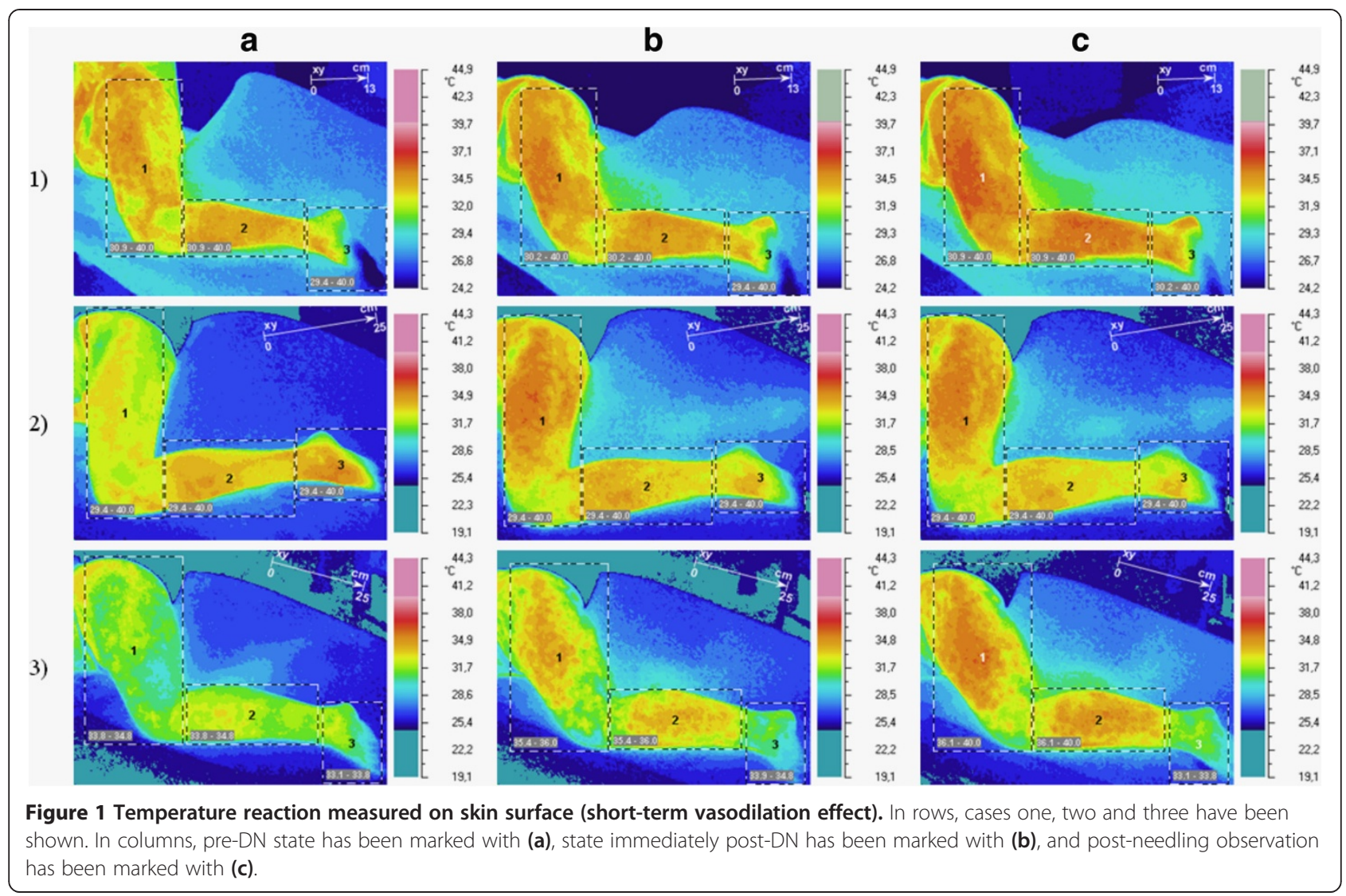

of myofascial pain syndrome among patients diagnosed with radicular or sciatica-like symptoms is possible. Although the findings presented in this paper are highly unique, the existence of autonomic phenomena (e.g. vasoconstriction in the area to which a trigger point refers pain) has already been suggested by Travell and Simons [3]. Some authors proved that intramuscular needling can induce vasodilation, but only locally [10]. Others tried to confirm the idea of Travell and Simons and confirmed the activation of the autonomic nervous system after nociceptive stimulation of latent TrPs (glutamate injections) in a non-symptomatic subject. However, they found IRT unable to detect vasodilation after $\mathrm{TrP}$ stimulation and they preferred to measure skin blood flow by laser Doppler flowmetry [11,12]. Quite interestingly, in other studies, the needle stimulation of the latent trigger point, skin and muscle point (acupuncture) provoked a decrease of skin temperature contrary to observations in this study [11-13]. In the observations presented in this study, the vasodilation effect increased during $\mathrm{DN}$, reached its highest post-DN and decreased afterwards. The vasodilation within the skin is provoked by Calcitonin Gen Related Peptide (CGRP) and Substance $\mathrm{P}$ release from $\mathrm{A} \delta$ nerve fibers and/or C-fibers $[5,14,15]$. Moreover, it is suggested that CGRP-related antidromic vasodilation may be important in clinical improvement of skeletal muscle blood flow produced by physical therapy, e.g. acupuncture [16]. Although the exact mechanism of DN remains unexplained, some authors propose that $\mathrm{DN}$ of active trigger points can stimulate sensory afferent $A \delta$ nerve fibers [5]. In the light of findings presented in this study, it seems that trigger point needle stimulation probably activated the sympathetic vasoconstriction mechanism and antagonized the primary afferent nerve-induced antidromic vasodilation. As mentioned above, some authors postulated the idea of the autonomic nervous system activity involvement among patients suffering from sciatica [2]. It seems that the cases presented in this paper support that idea. Although clinical signs of examined patients during bedside examination were typical of sciatica, the MRI results can spark some controversy. In the case of the patient with sciatica-like symptoms (case three), a disc-radicular conflict was found on the affected side. In the case of the patient with radicular pain diagnosis (case one), the MRI revealed a disc-radicular conflict on the opposite side (cross Lasegue was negative). Only in the case of patient two, both bedside examination and MRI results were typical of her condition. Nevertheless, no changes in nerve conduction or spinal reflex pathologies were found in any of the patients. It is now postulated that traditional diagnosis of sciatica patients should 


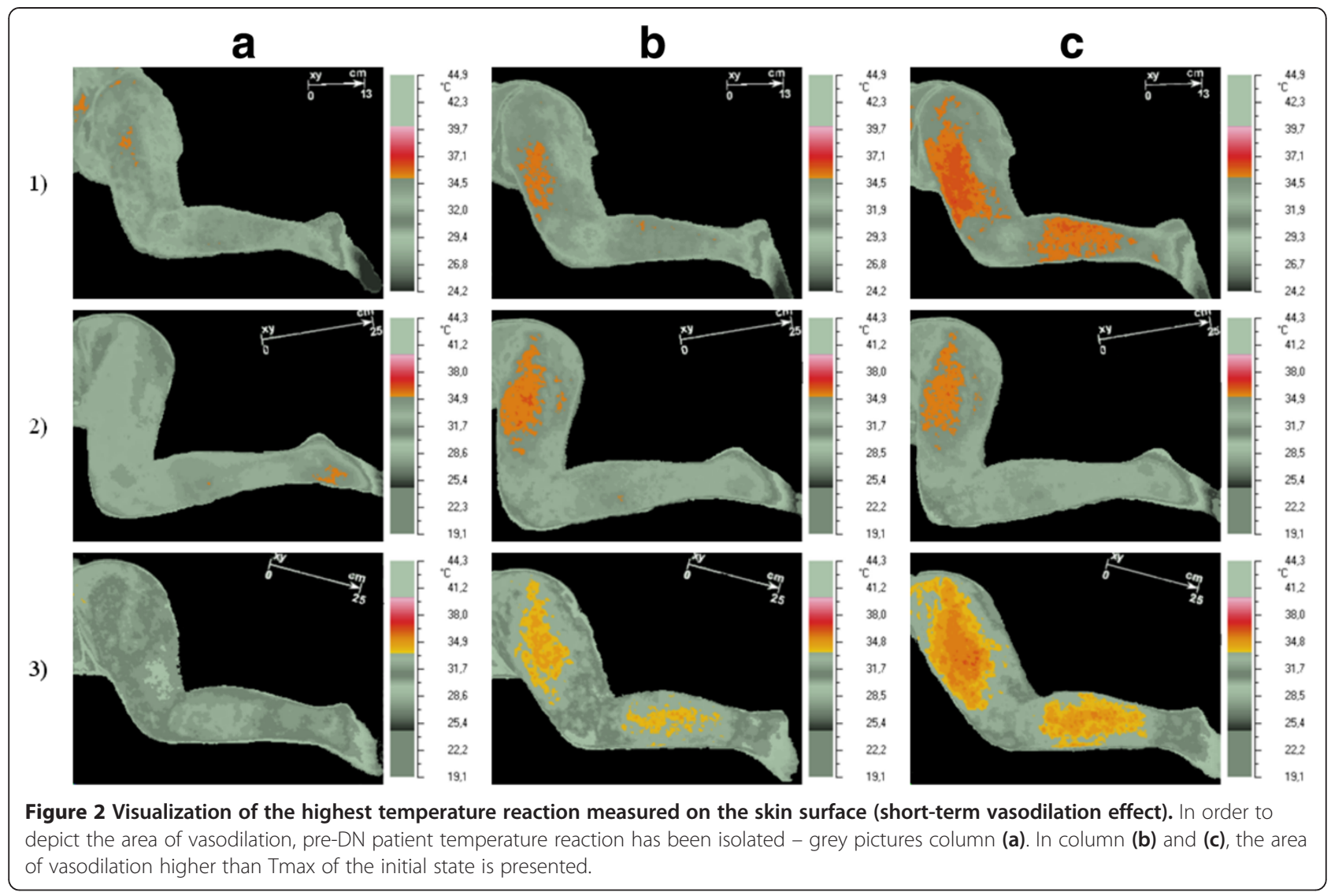

be followed by additional examinations, especially due to the fact that diagnostic accuracy of neurological signs and tests is unclear according to the literature on the subject [17]. Lasegue test is the only sign consistently reported to be sensitive for sciatica due to disc herniation, but its credibility is limited by its low specificity. Similarly, in MRI analysis, despite the fact that in majority of patients with pathology within disc area a strong correlation with pain in the lower limb is visible [18], sometimes it is possible to observe improvement with no changes concerning the disk [19], or the other way round: no improvement in spite of removing the disc protrusion or other reasons of nerve compression [20]. What is more, in some asymptomatic individuals, herniated nucleus pulposus occurred in MRI. There are also reports of patients suffering from confirmed disk pathology or with stenosis with apparent neural compromise, i.e. asymptomatic [21-23]. Apart from that, the laboratory blood test did not show any sign of inflammation. The situation in which laboratory blood tests, radiologic investigation or bedside examinations could not explain the source of the pain is typical of the neuropathic pain component. Because the examined patients did not undergo diagnostic tests evaluating neuropathic pain, it has not been proven that they suffered from neuropathic pain. However, in the light of findings presented in this study and existing theories stating that neuropathic pain patients suffer because of the activation of muscle fibers by sympathetic nerve activity [24], in the future it is important to evaluate the link between sciatica, neuropathic pain and trigger points. According to Travell, myofascial pain trigger points can mimic sciatica [3]. Myofascial pain and active trigger points of the gluteus minimus muscle were the only features to be found in all of the three examined cases. The aim of the study was not to deny the neurological diagnosis, however, clinical value of trigger points should be explored in the future. Further studies on a bigger group of sciatica patients co-diagnosed with myofascial pain are needed.

\section{Conclusion}

Conclusion: in the examined cases, trigger points related short-term vasodilation was recorded. Confirmation of these findings in a controlled, blinded study would indicate the existence of a link between the pain of sciatica patients (radicular or sciatica-like pain) and the activity of the autonomic nervous system. Further studies on a bigger group of patients are still needed.

\section{Consent}

Written informed consent was obtained from the three patients for publication of this case report and any 
accompanying images. A copy of the written consents are available for review by the Editor of this journal

\section{Additional file}

\section{Additional file 1: Short-term vasodilation effect during DN} completion and observation time (case one). The first three seconds picture the state of the patient pre-DN. In the third second, the DN of the first TrP can be observed. In the eighth second, the end of DN of the first $\operatorname{TrP}$ can be seen. In the eleventh second of the footage, the end of DN of the second TrP has been recorded. The final part of the footage (observation time, starting from eleventh and lasting until the fifteenth second) presents post-DN increase of vasodilation.

\section{Abreviations}

CGRP: Calcitonin Gen Related Peptide; CRP: C-reactive protein; DN: Dry needling; ESR: Erythrocyte sedimentation rate; GM: Gluteus minimus muscle; IRT: Infrared thermovision camera; LBP: Low back pain; MPS: Myofascial pain; MRI: Magnetic resonance imaging; NSE: Extensive neurological screening examination; Tavr: Average temperature; Tmax: Maximum temperature; TrPs: Trigger points; VAS: Visual-analogue scale.

\section{Competing interests}

The authors disclose no financial and personal relationships between themselves and others that might be perceived as biasing their work. No potential conflicts exist.

\section{Authors' contributions}

SE: conceived and designed the experiments, carried out the experiments, interpreted the data, drafted the manuscript, have given final approval of the version to be published; RM: conceived and designed the experiments, carried out the experiments, analysed and interpreted the data, drafted the manuscript, PW: performed the experiments, analysed and interpreted the data, AB: analysed and interpreted the data, SW: discussed the results and commented on the manuscript. All authors read and approved the final manuscript.

\section{Acknowledgements}

We thank all the study participants. Also, the authors gratefully acknowledge the critical reading and correction of this manuscript by Karolina Piwiec. The study was funded by the National Science Centre (ID number N N404 268339).

\section{Author details}

${ }^{1}$ Department of Rheumatology and Rehabilitation, Poznan University of Medical Sciences, Poznan, Poland. Department of Virtual Engineering, Poznan University of Technology, Poznan, Poland. ${ }^{3}$ University School of Physical Education, Department of Biomechanics, Poznan, Poland.

Received: 1 October 2013 Accepted: 4 September 2014 Published: 9 September 2014

\section{References}

1. Laplante BL, Ketchum JM, Saullo TR, DePalma MJ: Multivariable analysis of the relationship between pain referral patterns and the source of chronic low back pain. Pain Physician 2012, 15(2):171-178.

2. Freynhagen $\mathrm{R}$, Baron R: The evaluation of neuropathic components in low back pain. Curr Pain Headache Rep 2009, 13(3):185-190.

3. Simons DG, Travell J, Simons LS: Myofascial Pain and Dysfunction. The Trigger Point Manual, Volume 1. 2nd edition. Wiliams \& Wilkins: Baltimore; 1999.

4. Domingo A, Mayoral O, Monterde S, Santafé MM: Neuromuscular damage and repair after dry needling in mice. Evid Based Complement Alternat Med 2013, 2013:260806.

5. Dommerholt J: Dry needling - peripheral and central considerations. J Man Manip Ther 2011, 19(4):223-227.

6. Chen Q, Basford J, An KN: Ability of magnetic resonance elastography to assess taut bands. Clin Biomech 2008, 23:623-629.

7. Chen Q, Bensamoun S, Basford JR, Thompson JM, An KN: Identification and quantification of myofascial taut bands with magnetic resonance elastography. Arch Phys Med Rehabil 2007, 88:1658-1661.
8. Sikdar S, Shah JP, Gilliams E, Gebreab T, Gerber LH: Assessment of myofascial trigger points (MTrPs): a new application of ultrasound imaging and vibration sonoelastography, Proceeding of the 30th Annual International IEEE EMBS Conference; 2008 Aug 20-24. Vancouver, BC, Canada: Piscataway, NJ: IEEE; 2008:558.

9. Hubbard DR, Berkoff GM: Myofascial trigger points show spontaneous needle EMG activity. Spine 1993, 18:1803-1807.

10. Sandberg $M$, Lindberg $L G$, Gerdle B: Effects of acupuncture on skin and muscle blood flow in healthy subjects. Eur J App/ Physiol 2003, 90:114-119.

11. Zhang Y, Ge HY, Yue SW, Kimura Y, Arendt-Nielsen L: Attenuated skin blood flow response to nociceptive stimulation of latent myofascial trigger points. Arch Phys Med Rehabil 2009, 90(2):325-332.

12. Kimura $Y$, Ge HY, Zhang $Y$, Kimura M, Sumikura H, Arendt-Nielsen L: Evaluation of sympathetic vasoconstrictor response following nociceptive stimulation of latent myofascial trigger points in humans. Acta Physiol (Oxf) 2009, 196(4):411-417.

13. Agarwal-Kozlowski K, Lange AC, Beck H: Contact-free infrared thermography for assessing effects during acupuncture: a randomized, single-blinded, placebo-controlled crossover clinical trial. Anesthesiol 2009, 111(3):632-639.

14. Jänig W, Levine JD, Michaelis M: Interactions of sympathetic and primary afferent neurons following nerve injury and tissue trauma. Prog Brain Res 1996, 113:161-184.

15. Raja SN, Treede RD, Davis KD, Cambell JN: Systemic alpha-adrenergic blockade with phentolamine: a diagnostic test for sympathetically maintained pain. Anesthesiol 1991, 74:691-698.

16. Sato A, Sato Y, Shimura M, Uchida S: Calcitonin gene-related peptide produces skeletal muscle vasodilation following antidromic stimulation of unmyelinated afferents in the dorsal root in rats. Neurosci Lett 2000, 283(2):137-140.

17. Backup K: Clinical test for the musculoskeletal system. Examination- SignsPhenomena. 2nd edition. Stuttgart, Germany: Thieme; 2008

18. Porchet F, Wietlisbach V, Burnard B, Daeppen K, Villemure JG, Vader JP: Relationship between severity of lumbar disc disease and disability stores in sciatica patients. Neurosurg 2002, 50(6):1253-1259.

19. Garfin SR, Rydevik BL, Brown RA: Compressive neuropathy of spinal nerve roots. A mechanical or biological problem? Spine 1991, 16(5):162-166.

20. Stafford MA, Peng P, Hill DA: Sciatica: a review of history, epidemiology, pathogenesis, and role of epidural steroid injection in management. $\mathrm{Br}$ J Anaesthesia 2007, 99(4):461-473.

21. Boden SD, Davis DO, Dina TS: Abnormal magnetic resonance scans of the lumbar spine in asymptomatic subjects: a prospective investigation. J Bone Joint Surg [Am] 1990, 72(3):403-408.

22. Boss N, Semmer N, Elfering E, Schade V, Gal J, Zanetti M, Kislling R, Buchegger N, Hodler J, Main CJ: Natural history of individuals with asymptomatic disc abnormalities in magnetic resonance imaging. Spine 2000, 25(12):1484-1492.

23. Jensen MC, Brant-Zawadzki MN, Obuchowski N, Modic MT, Malkasian D, Ross JS: Magnetic resonance imaging of the lumbar spine in people without back pain. N Engl J Med 1994, 331(2):69-73.

24. Gunn CC: Neuoropathic myofascial pain syndrome. In Bonica's management of pain. Fishman SM, Ballantyne J, Ballantyne JC, Rathmell JP. 4th edition. Baltimore: Lippincott Williams \& Wilkins; 2010.

\section{doi:10.1186/1756-0500-7-620}

Cite this article as: Skorupska et al:: Intensive short-term vasodilation effect in the pain area of sciatica patients - case study. BMC Research Notes 2014 7:620. 